RICHARD SPITERI

\title{
MAURICE CIANTAR : DES AFFECTS ENGAGEANT LE TREFONDS
}

L'écriture idiosyncratique que pratique Ciantar implique la vie, privée ou publique, de l'auteur. Tantôt la vie de l'écrivain va à l'encontre d'une bourrasque, tantôt elle le laisse moisir au milieu du désert. Andrée Cariou, qui est la compagne de Ciantar pendant les années 1940, se sépare de lui, juste après avoir lu consternée Jacques Vorageolles, son premier roman ${ }^{1}$. Le succès de La Mongolique, son deuxième roman, entraîne presque la publication d'une traduction à Chicago ${ }^{2}$. Possibilité qui ne se matérialise finalement pas dans une Amérique puritaine. Mille jours à Pekin, journal que Ciantar a tenu dans la capitale chinoise au cours de la Révolution culturelle, est un pavé jeté dans le Paris de 1969 où les admirateurs de Mao sont légion. Ce geste ne tarde pas à créer pour Ciantar des ennemis ${ }^{3}$.

Frédéric Lordon préfère adopter la définition de Spinoza pour l'affect : l'affect est « la variation de puissance d'agir du corps et la formation d'idée qui résultent simultanément de cette affection $»^{4}$. Chez Ciantar, l'enfance, la famille, la société de son époque, autrement dit des années trente aux années soixante correspondant à la période de l'adolescence jusqu'à l'âge mûr de l'écrivain remodèlent en tant qu'affections son être, tout en offrant à l'écrivain l'occasion de s'assumer et d'explorer ses virtualités.

Compte tenu du fait que les textes littéraires de Ciantar s'imprègnent d'éléments autobiographiques, notre propos sera, dans un premier temps, d'examiner dans son cas une caractéristique fondamentale de l'être humain, les rapports entre les sexes. Ensuite nous verrons comment l'auteur, en proie à ses affects, s'adonne fréquemment à la dépense insensée de l'argent gagné, geste qui alarme non peu le lecteur bourgeois moyen. Enfin nous considérerons une technique de soi que Ciantar pratique tout le long de sa vie afin de tenir ses affects sous contrôle.

\footnotetext{
1 Voir Eric Séébold (éd.), Jacques Vorageolles et ses ombres: Maurice Ciantar 1915-1990, Tusson (Charente), Du Lérot, 2009, p. 463.

${ }^{2}$ Ibidem, p. 417.

${ }^{3}$ Ibidem, p. 500.

${ }^{4}$ Frédéric Lordon, La Société des affects : pour un structuralisme des passions, Paris, Points, 2015, p. 122.
} 


\section{La femme aux tendances terrestres}

Le premier couple évoqué par Ciantar dans Jacques Vorageolles, son premier roman autobiographique, consiste au vrai dans ses parents : Richard Vorageolles (Ciantar ne fait que changer le nom) et Marcelle Herber ${ }^{5}$ (c'est le nom de jeune fille de sa mère). Il y a au sein de ce couple des disparités criantes. D'abord l'âge des conjoints. L'année de leur mariage, 1913, Richard Ciantar a 47 ans tandis que la fiancée en a $21^{6}$. En outre il y a une énorme différence dans leur situation sociale. Bien que les deux soient arrivés assez récemment comme immigrés à Paris, Richard a le titre nobiliaire de « Paléologue » - nom de la dernière dynastie impériale de Byzance - il est propriétaire foncier et immobilier tout en pratiquant la peinture. En revanche, Marcelle n'est que la fille de petits commerçants. Le narrateur résume bien ce mariage : le père de Jacques Vorageolles « fut le grand seigneur, qui par bizarrerie sexuelle, se mésallia avec une fille de peu $»^{7}$.

Dans La Vie chronique, le journal de Ciantar qui a été publié posthumément, l'auteur donne un autre exemple de couples bizarres précisément parce que l'homme et la femme viennent d'aires géographiques très lointaines. Encore une fois l'affect décide de la formation de ces couples tout en bousculant des normes traditionnelles. En 1964, Ciantar s'installe à Tahiti où il travaille comme journaliste pour un quotidien de Papeete. Il observe le phénomène de militaires originaires de la métropole qui quittent leur épouse pour créer un foyer avec une Tahitienne même si celle-ci s'avère assez inculte dans les rapports sentimentaux ${ }^{8}$. D'après Ciantar, la raison en est que le Français, qui est dominé par son épouse, trouve à Tahiti le climat idoine pour s'imposer à l'autre sexe. Néanmoins, il suffit de très peu de temps pour que la Tahitienne réussisse à inverser la situation. De temps en temps l'auteur remarque une indigène, qui ne serait qu'une chambrière, prendre des grands airs avec son compagnon, officier galonné.

La différence d'âge du couple Vorageolles crée bientôt un grand gros problème pour le fils Jacques. Richard meurt laissant son fils de neuf ans vivre à l'ombre de Marcelle qui, devenue jeune veuve, se lance d'emblée à la recherche d'un deuxième époux. En fait, le petit Jacques a toujours souffert de la tendresse parcimonieuse que lui témoigne sa mère, carence qui a pour contrepartie, dans la

\footnotetext{
${ }^{5}$ Voir Maurice Ciantar, Jacques Vorageolles, Tusson (Charente), Du Lerot, 1987, p. 18.

${ }^{6}$ D'après «l'observation de la nationalité française $n^{\circ} 16911 »$ des archives de la préfecture de Paris, Marcelle est née en 1892. En ce qui concerne Richard, deux documents différents révèlent qu'il est né en 1866. En effet, sur le certificat de naissance de son fils Maurice, fait le 3 mars 1915, il déclare avoir quarante-neuf ans (voir Eric Séébold (éd.), Jacques Vorageolles et ses ombres, p. 439). De plus, l'édition du 3 mai 1924 du quotidien The Daily Malta Chronicle, annonce le décès de Richard à l'âge de 59 ans. Je ne trouve pas fiable la supposition de Jacques Vorageolles attribuant à Richard cinquante-six ans au moment de sa mort.

${ }^{7}$ Maurice Ciantar, Jacques Vorageolles, p. 21.

${ }^{8}$ Eric Séébold (éd.), Jacques Vorageolles et ses ombres, p. 73.
} 
trilogie Jacques Vorageolles, l'apparition autour de lui de dames d'âge mûr baignées de douceur ou faisant preuve au moins d'une attitude positive à son égard.

Un premier exemple figure au début de Jacques Vorageolles. L'été Richard emmène son épouse et son enfant à Monaco. Dans un palace attenant au casino, l'attention de l'enfant de huit ans est frappée par la beauté d'une dame à la fois charmante et coquette ${ }^{9}$. Jacques, soupirant trop précoce, fait sourire la dame de Monte-Carlo ainsi que ses amies. Ensuite il y a les années de l'éducation élémentaire. Expulsé du lycée Montaigne, Vorageolles finit au lycée Michelet où heureusement Mme Gourdeau, une institutrice vieillissante, adopte envers lui le rôle de grand-mère. Elle cajole le petit enfant au point que par deux fois il verse des larmes de bonheur ${ }^{10}$. Un autre épisode où Vorageolles jouit d'une protection féminine est celui qui se déroule à Barcelone pendant les premiers mois de la guerre civile. Vorageolles traverse la frontière franco-espagnole et s'associe aux recrues du POUM. Mais dès qu'il s'aperçoit de la superficialité de l'entraînement militaire, il s'effraie et décide de ne pas monter au front, quitte à devoir comparaître devant un conseil de guerre. Par miracle, il est libéré. Il doit attendre huit jours pour faire viser son passeport au Consulat de France. Heureusement une prostituée toulousaine lui trouve une piaule chez une vieille catalane et accepte également de lui payer le loyer ${ }^{11}$. Grâce à ces deux fées, Vorageolles réussit à s'évader de la Barcelone révolutionnaire devenue pour lui un enfer.

Par deux fois au moins, les femmes amoureuses de Vorageolles sont sensiblement plus âgées que lui au point qu'il joue le rôle de gigolo ${ }^{12}$. La guerre est la vraie cause de ces rapports sentimentaux singuliers. Les deux guerres mondiales de la première moitié $\mathrm{du} \mathrm{XX}^{\mathrm{e}}$ siècle où ont péri des centaines de milliers de militaires créent un déséquilibre démographique. Dans La Mongolique, Vorageolles âgé de dix-neuf ans fait la connaissance de Germaine Frande qu'il devine être quadragénaire. La bourgeoise parisienne l'invite dans son petit appartement cossu où elle lui montre des photos de soldats de la guerre de 1914 1918 et les lettres qu'ils lui envoyaient. Ils sont morts dans les tranchées. L'un en particulier lui a proposé le mariage ${ }^{13}$.

Le roman ...Et qu'on n'en parle plus! est consacré aux premiers mois de l'amour-passion que Ciantar et Andrée Cariou ont vécu ensemble. L'auteur prend note du jour exact où le couple se rencontre la première fois : c'est à Biarritz, le 8 juin 1940. Vorageolles est liftier à l'hôtel Miramar où la Dame aux danois - c'est l'appellation d'Andrée Cariou - vient loger. Le 8 juin c'est le lendemain de la

\footnotetext{
${ }^{9}$ Maurice Ciantar, Jacques Vorageolles, p. 27.

${ }^{10}$ Ibidem, pp. 87 et 94.

${ }^{11}$ Maurice Ciantar, La Mongolique, Paris, Gallimard, 1949, pp. 240-244.

${ }^{12}$ Dans Maurice Ciantar, ...Et qu'on n'en parle plus !, Tusson (Charente), Du Lerot, 1989, le terme « gigolo » a trois occurrences : pp. 72, 73, 129.

${ }^{13}$ Maurice Ciantar, La Mongolique, pp. 50, 105.
} 
bataille de Dunkerque. Le mari de la Dame aux danois est fait prisonnier par l'armée allemande et passera le reste de la guerre dans un stalag ${ }^{14}$. La guerre rapproche la Dame aux danois - qui de plus est mère d'une fille de six ans - à Vorageolles et cette famille recomposée vivra ensemble les années de l'occupation.

Chez Ciantar, les personnages féminins ont bonne intuition et leurs remarques sur Vorageolles sont justes. Les rapports intimes entre Vorageolles et Germaine Frande se terminent le jour où celle-ci découvre que son amant vole de l'argent dans son appartement. Plus tard Vorageolles s'enrôle dans le Légion étrangère. Avant son départ, Germaine accepte de venir lui dire adieu : "Au fond, tu n'es pas mauvais, lui dit-elle. Il t'a manqué un guide. Tu as trop été livré à toi-même $»^{15}$.

Vorageolles s'estime injustement traité par Marcelle, sa mère. Quand la possibilité de mariage entre Vorageolles et Lydie - une étudiante - se profile à l'horizon, Marcelle vient discuter avec le père de la fiancée. Mais le doux projet s'évanouit, surtout lorsque Marcelle raconte au père de la fiancée que son fils « est un dépensier, un coureur de jupons... Que toute sa vie [il ne serait] qu'un bohème $\rangle^{16}$. En effet Vorageolles n'a pas de métier. Plus tard il modifie l'opinion qu'il se fait de sa mère. Il la remercie de lui avoir permis, à l'âge de 17 ans, de quitter le foyer et de se griser de liberté ${ }^{17}$.

\section{Le goût de la dilapidation}

L'insertion dans le monde se fait au moyen du travail. Comme dit Georges Bataille: "Le travail a déterminé l'opposition du monde sacré et du monde profane $^{18}$. » Vorageolles adolescent est invité par son beau-père Marcel G. à faire ses débuts comme apprenti dans une de ses boutiques, celle du boulevard Blanqui près des Gobelins. Mais les manquements de professionnalisme de Vorageolles sont manifestes. Tantôt lui et un commis empochent l'argent d'une vente, tantôt il s'absente et revient à la boutique juste avant l'heure de fermeture ${ }^{19}$. Une autre fois, pour plaire à l'oncle de Vorageolles, un certain M. Lemoine « représentant de fabriques $^{20} \gg 1$ 'emploie dans son entreprise. La première tâche consiste en balayage des bureaux. Ensuite Vorageolles fait surtout de la manutention. Mais après qu'il perçoit le premier salaire mensuel il ne se fait plus voir dans l'entreprise.

À partir de l'été 1944, Ciantar exerce la profession de journaliste. Il est d'abord " chef de service de presse du ministre de Ravitaillement ${ }^{21}$. Ensuite il

\footnotetext{
${ }^{14}$ Maurice Ciantar, ...Et qu'on n'en parle plus !, p. 116.

${ }^{15}$ Maurice Ciantar, La Mongolique, p. 121.

${ }^{16}$ Ibidem, p. 188.

${ }^{17}$ Ibidem, p. 285.

${ }^{18}$ G. Bataille, L'Érotisme, Paris, Minuit, 2014, p. 123.

${ }^{19}$ Voir Maurice Ciantar, Jacques Vorageolles, pp. 330, 357.

${ }^{20}$ Maurice Ciantar, La Mongolique, p. 20.

${ }^{21}$ Eric Séébold (éd.), Jacques Vorageolles et ses ombres, p. 455.
} 
rapporte les nouvelles parlementaires au Populaire et à Le Pays. Il est chargé des revues des spectacles dans Combat et dans Paris Jour. Il collabore à deux chaînes de radio : secrétaire de rédaction à Europe ${ }^{\circ} 1$ et éditorialiste à France Culture. Lors de son départ à la retraite il est journaliste à l'hebdomadaire Le Point.

Cette profession ne procure pas à Ciantar la satisfaction tant attendue. Une note qu'il écrit sur un cahier en septembre 1964 dévoile son avenir morne : «Journaliste depuis bientôt vingt-deux ans... et cela me conduit par les routes merveilleuses du rien ${ }^{22}$ !»

Vorageolles qui est inapte au travail dans le petit commerce ne tarde pas à jeter sa gourme. Marcelle émancipe son fils lorsque celui-ci a dix-huit ans. Après avoir vendu une propriété ayant appartenu à Richard, elle accorde à son fils une somme d'argent. Vorageolles se permet des vacances à Barcelone où la première nuit on l'amène écouter des travestis chanter dans un beuglant ${ }^{23}$. Après trois mois de fainéantise, Vorageolles, ayant tout dépensé, rentre à Paris.

Un des thèmes principaux des œuvres tardives de Ciantar est le jeu au casino, thème qui domine dans Le Journal d'Edmond, autre roman autobiographique. Cette fois, l'alter ego de Ciantar s'appelle Edmond tandis qu'Andrée Cariou est Maya. Sur fond de climat morbide régnant à Nice pendant l'occupation, un certain Claude T. invite Edmond à l'accompagner au casino de Monte-Carlo ${ }^{24}$. Edmond est ravi de cette expérience. À Paris, quelques années plus tard, après une dispute avec un rédacteur-en-chef, Edmond démissionne de son emploi de journaliste. Cette déconvenue conjuguée avec l'infidélité de Maya de laquelle il se sépare, le persuade de s'installer plutôt à Nice pour y vivre du jeu au casino. Le Journal d'Edmond montre toujours le personnage éponyme qui, dans les casinos, coudoie un joueur expert ; le roman traite en effet une initiation au jeu de la roulette.

Dans Maman Salope le narrateur décrit l'exaltation qu'il éprouve après voir gagné des sommes importantes à la roulette. Le texte consiste en une suite de noms propres ou nom de pays associés au luxe. «Une fois sorti de l'usine, les Borgia! La gonzesse-taximètre. Sans complication. Tout! Victuailles ! Arabie Saoudite ! Hong-Kong! - Onassis, quoi ? - N'exagérons rien, Eusèbe $»^{25}$. Mais autant les triomphes procurent à Ciantar un ravissement divin autant les pertes le plongent dans une morosité abyssale. Les notations dans le journal de Ciantar pour le mois d'août 1962 révèlent qu'au casino de Cannes, par deux fois, il jette deux sommes d'argent importantes par la fenêtre. La séquence commence par les regrets exprimés par l'auteur d'avoir perdu stupidement 450000 AF. Reprenant du courage, il remet les pieds dans le casino où soir après soir il recommence à gagner $^{26}$. Son ambition: récupérer la somme perdue. Les gains montent et

${ }^{22}$ Ibidem, p. 72.

${ }^{23}$ Maurice Ciantar, La Mongolique, p. 28.

${ }^{24}$ Maurice Ciantar, Le Journal d'Edmond, Tusson (Charente), Du Lerot, 2001, p. 71.

${ }^{25}$ Maurice Ciantar, Maman Salope, Tusson (Charente), Du Lérot, 1985, p. 51.

${ }^{26}$ Voir Eric Séébold (éd.), Jacques Vorageolles et ses ombres, pp. 48, 53, 54, 57, 59, 60. 
finalement ils dépassent la somme fatidique. Mais juste avant de rentrer à Paris, au cours d'une soirée maudite, il perd tous les derniers gains !

Pour revenir à la vie sentimentale de Ciantar, la première fois où il faillit créer une famille c'est avec Andrée Cariou. Il raconte cette expérience dans ...Et qu'on n'en parle plus! qui est le troisième roman de la trilogie Jacques Vorageolles. À Nice, le $1^{\text {er }}$ février 1942, Vorageolles et Maya ont un enfant appelé Lélian-Wilde - le premier terme est le prénom familier attribué à Paul Verlaine - qui meurt dix-huit jours plus tard ${ }^{27}$.

Ciantar reste célibataire sa vie durant. Pour autant son Éros ne s'endort pas, loin s'en faut. Le travail sur le langage dont l'auteur distille les effets poétiques neutralise d'habitude la trivialité de l'action. Les textes décrivent au moins deux épisodes d'érotisme collectif qui ont lieu à Paris dans «le Jardin aux deux métamorphoses » avant ou après le crépuscule ${ }^{28}$. Dans le premier épisode, Edmond s'y rend avec une "rédactrice » de ministère et, dans le deuxième, Dubitatif s'y trouve déjà avec la «gouvernante d'un vieux monsieur ». Les deux narrateurs appellent « arabesques » les deux scènes.

Les participants passifs ou actifs de ces scénarios se déroulant au sein d'un jardin public sont désignés non par leur nom mais plutôt par le métier qu'ils exercent. Outre la rédactrice de ministère et la gouvernante, surviennent un pharmacien et son épouse, un plombier, deux employés de banque, un cheminot et un certain type surnommé « wagon-lit». Des bourgeois et des prolétaires prennent part aux ébats crépusculaires, ce qui, d'après le narrateur d'Un Bouton dans la rue, démontre qu'Éros brasse les classes sociales si ce n'est que pour une brève durée ${ }^{29}$. $\mathrm{Vu}$ l'heure tardive, les participants apparaissent surtout comme des ombres. En désignant une silhouette qui s'approche, la gouvernante demande à Dubitatif assis auprès d'elle : " - Vous le connaissez ? - Personne ici ne se connaît mais tous se reconnaissent $»^{30}$. Ceci implique qu'aussitôt terminée, l'orgie livre Dubitatif à sa solitude ordinaire.

\section{Une technique de soi}

Examinons maintenant la place que Ciantar occupe dans la société. Deux affects presque contraires déterminent son comportement dans ce domaine: la tendance grégaire d'une part et son goût pour la flânerie d'autre part.

Dans La Mongolique, le narrateur décrit l'éveil de Vorageolles aux enjeux politiques brûlants qui déchirent les Français pendant les années trente. Vorageolles n'a que dix-neuf ans, il est précepteur d'enfants de familles de l'île

\footnotetext{
${ }^{27}$ Maurice Ciantar, ...Et Qu'on n'en parle plus !, pp. 172, 178.

${ }^{28}$ Maurice Ciantar, Le Journal d'Edmond, pp. 105, 108-109 et Maurice Ciantar, Un Bouton dans la rue, Tusson (Charente), Du Lérot, 1983, pp. 49-55.

${ }^{29}$ Maurice Ciantar, Le Journal d'Edmond, p. 106.

${ }^{30}$ Maurice Ciantar, Un Bouton dans la rue, p. 54.
} 
Saint-Louis mais le reste de son temps il déambule dans Paris. Le 20 janvier 1934 éclate le scandale Stavisky ${ }^{31}$. L'après-midi du 6 février, Vorageolles s'unit à la foule en colère qui marche vers le palais Bourbon. Après la tuerie lamentable sur la place de la Concorde, Vorageolles décide de militer au sein de la gauche, activité qui le verra deux ans plus tard participer à la guerre d'Espagne.

Pourtant membre d'organismes politiques, il court d'une désillusion à l'autre. La SFIO à laquelle il adhère, accueille deux socio-démocrates allemands évadés des prisons hitlériennes sans leur accorder de l'aide matérielle ${ }^{32}$. Tenté par le trotskisme, Vorageolles se met à assister aux réunions hebdomadaires de sa section où il est abasourdi par le spectacle de camarades français applaudissant des Israélites venus de Pologne qui vilipendent l'esprit «petit bourgeois» de la France $^{33}$. Enfin, au cours de l'été 1936, arrivé à Barcelone insurgée, il rejoint sa colonne du POUM. Mais dans la capitale catalane, la révolution prolétarienne dégénère. Le comble est atteint lorsque l'entraînement militaire de la colonne du POUM est confié à deux Italiens refugiés à Paris où ils ont travaillé «dans l'épicerie $»^{34}$. À partir de ce moment, Vorageolles ne songe qu'à rentrer en France.

Puisque Vorageolles adolescent préfère mener une vie de bohème au lieu de travailler dans une boutique ou une petite entreprise, force est de constater que la marginalité qu'il embrasse rehausse sa vigueur morale. Contrariant sa mère, Vorageolles ne s'inscrit pas en faculté de droit, mais adore traîner dans le Quartier Latin. Son lieu de prédilection est le Bar de la Sorbonne où le BarSo, comme les étudiants l'appellent familièrement. En fait, c'est un restaurant russe : le personnel consiste en réfugiés fuyant la révolution bolchévique. Vorageolles s'y installe du matin jusqu'à midi et de quatorze heures à seize heures juste pour se sentir proche des étudiants qui y étalent leurs déboires ou éclatent de rire ${ }^{35}$. Pendant l'intervalle Vorageolles flâne dans les parages. A vrai dire, des vieux messieurs cultivés fréquentent aussi le BarSo. Un des jours les plus heureux pour Vorageolles est celui où il noue conversation avec Daille, un étudiant, qui bientôt le présente à ses collègues $^{36}$.

Manquant souvent d'argent, Vorageolles traverse Paris à pied en tous sens ${ }^{37}$. C'est la vie de Bohème. La flânerie chez Ciantar relève des techniques de soi que Foucault définit de la manière suivante : des «procédures [...] prescrites aux individus pour fixer leur identité, la maintenir ou la transformer [...] grâce à des

\footnotetext{
${ }^{31}$ Maurice Ciantar, La Mongolique, p. 76.

32 Ibidem, pp. 149-150.

${ }^{33}$ Ibidem, p. 153.

${ }^{34}$ Ibidem, p. 222.

${ }^{35}$ Maurice Ciantar, Jacques Vorageolles, pp. 354-356, 359-360.

${ }^{36}$ Ibidem, p. 364.

${ }^{37}$ Maurice Ciantar, La Mongolique, pp. 60, 280.
} 
rapports de maîtrise de soi sur soi ${ }^{38}$. » L'importance de la flânerie est telle qu'elle le guide dans le choix d'une idéologie politique. Bien que le soir du 6 février 1934, Vorageolles se mêle aux manifestants de droite, peu après il leur tourne le dos pour une raison curieuse. Il craint que les Ligues n'endommagent un système politique français qui, tout faible qu'il puisse être, continue à protéger des libertés fondamentales comme, par exemple, la liberté de s'adonner à la flânerie ${ }^{39}$.

Surtout la flânerie inspire à Vorageolles le désir de lire, passion qu'il assouvit en passant de longues heures à la Bibliothèque Sainte-Geneviève. À remarquer que dans Jacques Vorageolles et La Mongolique figurent des strophes d'Oscar Wilde, de Verlaine et de Musset ${ }^{40}$. En outre, le premier roman contient sur la flânerie une référence intéressante. Encore une fois, la société met en demeure le jeune protagoniste d'ignorer l'individualité et d'opter pour l'uniformité. Vorageolles prend une décision importante, celle de devancer l'âge du service militaire. Dans une lettre à sa mère, il exprime des doutes à propos d'une carrière sous les drapeaux. " Je repense, lui écrit-il, à mon existence flâneuse, débraillée, dont les bienfaits (mais oui...) se feront quelque jour sentir » ${ }^{41}$. Petit à petit le flâneur se transforme en poète. Dans les deux romans indiqués, Ciantar inclut au moins le texte de trois de ses poèmes: "Souffrances inconscientes de l'enfant», « Mougdagaloune» et « Les Pauvres hommes que nous sommes » ${ }^{42}$. Quand même il faudrait souligner que peu de la production poétique de Ciantar des années trente a survécu. L'écrivain révisait ses textes de manière obsessionnelle ce qui fait que périodiquement il déchirait des manuscrits. Justement, le 3 septembre 1939, Vorageolles porte une serviette contenant un grand nombre de ses poèmes. Mais après avoir entendu à la radio la nouvelle de la déclaration de guerre de la Grande Bretagne à l'Allemagne, il se rend au jardin du Luxembourg où il jette au vent toutes les bonnes feuilles ${ }^{43}$.

Jusqu'à un âge avancé, Ciantar a continué à pratiquer la marche dans Paris et sa banlieue. Nous disposons là-dessus du témoignage de Jean Guenot : «Éternel beau gosse à soixante-dix ans passés, Maurice Ciantar [se promenait] quotidiennement sur le boulevard Bineau, à Neuilly, et jusqu'au restaurant chinois de la rue Louise-Michel à Levallois [...] ${ }^{44}$.

En conclusion, les affects se présentent comme un appel remontant du tréfonds de l'être humain pour résonner dans la conscience. Ils induisent à la

\footnotetext{
${ }^{38}$ Michel Foucault, «Subjectivité et vérité », in Dits et Écrits IV (1980-1988), Paris, Gallimard, 1994, p. 213.

${ }^{39}$ Maurice Ciantar, Le Journal d'Edmond, p. 31.

${ }^{40}$ Maurice Ciantar, Jacques Vorageolles, p. 385 et Maurice Ciantar, La Mongolique, pp. 135, 272.

${ }^{41}$ Maurice Ciantar, Jacques Vorageolles, p. 421.

42 Ibidem, p. 28, 43 et Maurice Ciantar, La Mongolique, pp. 286-288.

${ }^{43}$ Ibidem, p. 299.

${ }^{44}$ Jean Guenot, " Maurice Ciantar hussard à pied », in Eric Séébold (éd.), Jacques Vorageolles et ses ombres, p. 487.
} 
réappropriation de la liberté, donnent au corps l'impulsion d'agir dans la société. Les protagonistes dans les romans et récits de Ciantar réalisent des transgressions - chères à Georges Bataille. Sans complexes, dans le contexte de l'après-guerre, le jeune Vorageolles joue le rôle de gigolo auprès de dames plus âgées. Dubitatif participe à des arabesques tout en négligeant de fonder un foyer. Edmond voudrait faire du jeu au casino sa véritable profession, ambition généralement perçue comme choquante. Pour revenir à Vorageolles, il se désintéresse éventuellement des organismes politiques leur préférant des errances sur les boulevards.

Même structure de comportement que Spinoza définit par le conatus et Foucault par la technique de soi. Les affections amènent les personnages de Ciantar à connaître leur soi par soi, les propulsent vers l'affirmation d'eux-mêmes parmi les autres.

\section{BIBLIOGRAPHIE}

Archives de la préfecture de Paris (Pré Saint-Gervais), observation nationalité française $\mathrm{n}^{\circ} 16911$ : Marcelle Ciantar née Herber.

BATAILLE, Georges, L'Érotisme [1957], Paris, Minuit, 2014.

CIANTAR, Maurice, Un Bouton dans la rue, Tusson (Charente), Du Lérot, 1983.

CIANTAR, Maurice, ...Et qu'on n'en parle plus ! [1952], Tusson (Charente), Du Lérot, 1989.

CIANTAR, Maurice, Jacques Vorageolles [1947]. Note de l'éditeur Jean-Paul Louis et préface de Jacqueline Lemercier, Tusson (Charente), Du Lérot, 1987.

CIANTAR, Maurice, Le Journal d'Edmond. Préface d'Eric Séébold, Tusson (Charente), Du Lérot, 2001.

CIANTAR, Maurice, Maman Salope [1973], Tusson (Charente), Du Lérot, 1985.

CIANTAR, Maurice, La Mongolique, Paris, Gallimard, 1949.

CIANTAR, Maurice, Mille jours à Pékin [1969], Tusson (Charente), Du Lérot, 1990.

The Daily Malta Chronicle, Malte, La Valette, 1924, 3 mai.

FOUCAULT, Michel, Dits et écrits IV (1980-1988), Paris, Gallimard, 1994.

LORDON, Frédéric, La Société des affects : pour un structuralisme des passions, Paris, Seuil, 2015.

SEEBOLD, Eric (éd.), Jacques Vorageolles, Jacques Vorageolles et ses ombres : Maurice Ciantar (1915-1990), Tusson (Charente), Du Lérot, 2010.

ZAC, Sylvain, "Spinoza », in Histoire de la philosophie II. Sous la direction d'Yvon Belaval, Paris, Gallimard, 1981, pp. 452-482.

\section{MAURICE CIANTAR: AFFECTS FROM THE DEPTHS (Abstract)}

In this paper I intend to show how affects that come to the surface in Ciantar's writings mark the relations between the sexes and account for the characters' controversial bent on how they spend their energy. Finally, the text reveals a clash between the urge to join the crowd and the call of the 
Bohemian way of life. Ciantar's novels reveal the age gap between a man and a woman forming a couple and stress acute differences in their social status. In the Jacques Vorageolles trilogy, on two occasions, the protagonist ends up with an older female partner and earns the reputation of being a gigolo. Vorageolles' brief experience of fatherhood is cut short due to the passing away of his prematurely born child. In order to recover, , the male protagonist in Ciantar's texts remains a bachelor, takes part in orgies and, childless, faces courageously the onset of old age. Gambling is practiced as a profession by the author and comes to the fore in the later texts. While still an adolescent, Vorageolles joins first the SFIO, then a Trotskyite group, before heading for Barcelona at the outbreak of the civil war where, soon, reality dampens his revolutionary enthusiasm. As from now on, Ciantar turns his back to the crowd, preferring definitely to stroll endlessly along the Paris boulevards: an activity which is equivalent to Foucault's technique of the self.

Keywords: Maurice Ciantar, Bohemian, promiscuity, squander, technique of the self.

\section{MAURICE CIANTAR: AFECTE PROFUNDE (Rezumat)}

Studiul de faţă îşi propune să arate felul în care sentimentele predominante din scrierile lui Ciantar marchează relaţia dintre sexe şi explică pornirile controversate ale personajelor de a-şi consuma energia. În cele din urmă, textul relevă divergenţa dintre două tendinţe, aceea de a se alătura mulţimii şi aceea de a îmbrăţişa un mod de viaţă boem. Romanele lui Ciantar vorbesc despre diferenţele de vârstă dintre bărbaţi şi femei în interiorul cuplului şi pun accent pe divergenţele acute dintre sexe în privinţa statutului social. Protagonistul din trilogia Jacques Vorageolles ajunge în două rânduri să aibă o parteneră mai în vârstă ca el, câştigându-şi, astfel, reputaţia de gigolo. Scurta experienţă paternă a lui Vorageolles este întreruptă de moartea copilului său prematur. Pentru a depăşi situaţia, protagonistul din textele lui Ciantar rămâne burlac, ia parte la orgii, apoi, rămas fără copil, înfruntă curajos perspectiva bătrâneţii. Practicarea jocurilor de noroc constituie o profesiune pentru autor şi apare în prim plan în scrierile sale ulterioare. Adolescent fiind, Vorageolles aderă mai întâi la SFIO, apoi la o grupare troţkistă, înainte de a se îndrepta către Barcelona la izbucnirea războiului civil, unde, în scurt timp, realitatea îi va tempera entuziasmul revoluţionar. De acum înainte, Ciantar va întoarce spatele colectivităţii, preferând definitiv să străbată în lung şi în lat străzile Parisului, o activitate echivalentă cu tehnica sinelui descrisă de Foucault.

Cuvinte-cheie: Maurice Ciantar, boem, promiscuitate, risipă, tehnica sinelui. 